\title{
Revisão Sistemática Sobre a Eficácia de Intervenção Fisioterapêutica em Comparação com a Terapia Vibratória sobre o Equilíbrio Postural e Marcha em Indivíduos com Doença de Parkinson Systematic Review on the Effectiveness of Physiotherapeutic Intervention in Comparison With Vibratory Therapy on Postural Balance and Gait in Parkinson's Disease
}

\author{
Marcos Vinicius Carvalho Guimarães ${ }^{\mathrm{a}}$; Marcio Luis dos Santos*a
}

\begin{abstract}
${ }^{a}$ Universidade Anhanguera de São Paulo, Programa de Pós-Graduação Stricto Sensu em Biotecnologia e Inovação em Saúde. SP, Brasil.
\end{abstract}
*E-mail: marcio.1.santos@anhanguera.com

\begin{abstract}
Resumo
As alterações na marcha e no controle postural de pacientes com doença de Parkinson (DP) são incapacitantes, levando a alta incidência e prevalência de quedas e diminuição da qualidade de vida. A intervenção física, por meio de exercícios direcionados, tem sido apontada como um dos meios mais eficazes de reabilitação do controle postural e marcha. O Treino para Recuperação de Perturbação Externa (TRPE) temse mostrado um método promissor para diminuir as alterações de postura e marcha em diversas populações. O treinamento combinado de equilíbrio, associado ao biofeedback da Estimulação Mecânica vibratória (EMV), demonstrou diminuir a oscilação de tronco em indivíduos com DP, melhorando o controle postural. Apesar das intervenções apresentarem características diferentes e promissoras em estudos isolados, a potencialização dos efeitos com as intervenções propostas poderão trazer um direcionamento para elaboração de um protocolo inovador. Sendo assim, o objetivo desta pesquisa foi realizar uma revisão sistemática de ensaios clínicos aleatorizados sobre a eficácia do TRPE e/ou EMV, no tratamento do equilíbrio postural e marcha em indivíduos com DP. As buscas foram realizadas em quatro bases de dados eletrônicas: Medline, SciELO, PEDro e Central. De 303 estudos identificados inicialmente, 16 foram incluídos. Com base nos estudos analisados, demonstrou-se que o treino para recuperação de perturbação externa e/ou a estimulação mecânica vibratória, favorecem a melhora do desempenho da marcha e no tratamento da instabilidade postural em indivíduos com DP. Entretanto, existe um número reduzido de estudos controlados, aleatorizados, sobre ambas as intervenções em pacientes com DP.
\end{abstract}

Palavras-chave: Doença de Parkinson. Treino de Recuperação de Perturbação Externa. Estimulação Mecânica Vibratória.

\begin{abstract}
Changes in gait and postural control in patients with Parkinson's disease (PD) are disabling, leading to a high incidence and prevalence of falls and decreased quality of life. Physical intervention, through targeted exercises, has been identified as one of the most effective means of rehabilitation of postural and gait control. Training for Recovery of External Perturbation (TREP) has been showing to be a promising method for reducing posture and gait changes in different populations. The combined balance training associated with the biofeedback of Mechanical Vibratory Stimulation (MVS) has shown to decrease trunk oscillation in individuals with PD, improving postural control. Although the interventions present different and promising characteristics in isolated studies, the potentiation of the effects with the proposed interventions may bring a direction for the elaboration of an innovative protocol. Therefore, the purpose of this research was to conduct a systematic review of randomized clinical trials on the effectiveness of TREP and/or MVS in the treatment of postural balance and gait in individuals with PD. The searches were carried out in four electronic databases: Medline, SciELO, PEDro, and Central. Of the 303 studies initially identified, 16 were included. Based on the studies analyzed, it has shown that TREP and/or MVS favors the improvement of gait performance and the treatment of postural instability in individuals with PD. However, there is a small number of controlled, randomized studies on both interventions in PD patients.
\end{abstract}

Keywords: Parkinson's Disease. External Disturbance Recovery Training. Mechanical Vibration Stimulation.

\section{Introdução}

A doença de Parkinson - DP é uma doença neurodegenerativa, que se torna mais comum, com o decorrer da idade, sendo o marcador patológico mais importante a degeneração seletiva de neurônios dopaminérgicos na parte compacta da substância negra (SILVA et al., 2016). Estudos recentes apontam a presença neurotóxica de inclusões chamadas corpos de Lewy formadas por agregados proteicos de alfa-sinucleína (LENT, 2010).

A DP está frequentemente associada a um conjunto de sintomas não motores, como distúrbios de fala, sono, olfato e cognitivos, sendo acompanhados ou podendo preceder os sintomas motores (SILVA et al., 2016). Os sintomas motores relacionados a DP são: tremor de repouso, rigidez, bradicinesia e instabilidade postural (JANKOVIC, 2008). A falta de equilíbrio postural, normalmente ocorre em estágios avançados da DP, sendo um dos sintomas mais incapacitantes, na medida em que prejudica atividades de vida diária, com consequente aumento do risco de quedas (SCHONEBURG et.al., 2013). O controle postural depende da ação constante de dois mecanismos: o ajuste postural antecipatório e reativo (LEE et al., 1995). O controle postural reativo ou compensatório, foco do presente estudo, é responsável pela manutenção do equilíbrio postural durante perturbações 
externas e imprevisíveis, como durante um empurrão, tropeço ou qualquer perturbação corporal que não pode ser prevista (LAESSOE et al., 2008).

Especificamente, estudos indicam que as respostas posturais reativas de indivíduos com DP são atrasadas e menores (MANCINI et al., 2008; HELY et al., 2008) em comparação com idosos sadios. Além disso, os indivíduos com DP acabam compensando, com o sistema visual, o comprometimento proprioceptivo que reflete em desordens no equilíbrio postural, marcha e queda funcional (KLOCKGETHER et al., 1995; AHLSKO, 2011). Apesar da eficácia das medicações, como levodopa (droga mais potente), para melhorar os sintomas motores, como tremor e bradicinesia, a instabilidade postural não é beneficiada pela ação medicamentosa. De fato, estudos indicam que a levodopa piora a instabilidade postural, aumentando o risco de quedas de indivíduos com DP (CRENNA et al., 2006; GEORGE et al., 2015). Por outro lado, a atividade física direcionada tem mostrado efeitos positivos para melhora das desordens do equilíbrio postural com consequente diminuição do risco de quedas (SHEN et al., 2014; MANSFIELD et al., 2014).

Estudos em animais e humanos indicam que a atividade física direcionada proporciona uma melhora do controle postural e retarda a progressão da doença em indivíduos com DP (MORRIS et al., 2010; MAK et al., 2017). O exercício exerce um efeito neuroprotetivo, aumentando a produção de fatores de crescimento, Brain-derived neurotrophic factor (BDNF) e glial cell line- derived neurotrophic factor - GDNF, atenuando o dano dopaminérgico neuronal, com potencial de reduzir a inflamação celular e o estresse oxidativo, promovendo a neurogênese endógena (TAJIRI et al., 2010; TSOU et al., 2015). Assim, a prática de atividade física é promissora no tratamento das desordens de equilíbrio postural na DP. Em consonância com a atividade fisica, a estimulação mecânica vibratória alternada nos músculos do tronco tem sido utilizada para fins terapêuticos na DP, proporcionando uma melhora no controle do tronco e da marcha (VALKOVIC et al., 2006; DE NUNZIO et al., 2010).

A estimulação mecânica vibratória (EMV) é um método em que o estímulo vibratório é aplicado no tendão do músculo, ventre muscular ou na orientação das resultantes da força muscular (COUTO et al., 2012, DRUMMOND, 2012). Segundo Nanhoe-Mahabier et al. (2012), o treinamento combinado de equilíbrio associado ao biofeedback da EMV, demonstrou diminuir a oscilação de tronco em indivíduos com DP, melhorando o controle postural. Entretanto, no âmbito da reabilitação, a atividade física precisa ser estruturada de forma que o paciente com DP tenha uma aprendizagem eficiente para que consiga transferir o que aprende nas sessões de fisioterapia para suas atividades de vida diária (ABBRUZZESE et al., 2009).

O treino para recuperação de perturbação externa - TRPE é uma intervenção que incorpora perturbações, repetidas e inesperadas, externas, para evocar reações rápidas de equilíbrio, permitindo ao indivíduo treinar o controle postural reativo utilizando prática específica (MAKI et al., 2005). Estudos recentes encontraram efeitos positivos no TRPE em idosos sadios e indivíduos com DP, reduzindo o risco de quedas (SMANIA et al., 2010; SHEN et al., 2012). Portanto, as evidências científicas apontam que a combinação de um treinamento baseado em perturbações ou em EMV, podem melhorar a estabilidade postural em indivíduos com DP (VOLPE et al., 2014; LANDERS et al., 2016).

\section{Desenvolvimento}

\subsection{Metodologia}

\subsubsection{Tipo de estudo}

O presente estudo compreende uma revisão sistemática realizada de acordo com as orientações da lista de verificação e declaração dos Principais Itens para Relatar Revisões Sistemáticas e Meta-análises PRISMA (LIBERATI et al., 2009). A Revisão Sistemática foi organizada a partir dos seguintes tópicos: elaboração de uma questão norteadora para o método de busca, utilizando a estratégia PICO [acrônimo para Paciente, Intervenção, comparação e "Outcomes" (desfecho/resultado)], no qual P (população/participantes) foi representado por indivíduos com Doença de Parkinson; I (intervenção/procedimento), por intervenções, tais como, TRPE e EVM; C (Comparação), com a fisioterapia convencional (exercícios resistidos e/ou alongamentos) $\mathrm{O}$ (resultado) representou o sucesso do gerenciamento na redução do número de quedas, na melhora do equilíbrio postural e marcha em indivíduos com DP.

\subsubsection{Estratégias de busca}

A estratégia de busca e os critérios de inclusão e exclusão para os estudos selecionados foram avaliados por dois revisores de forma independente (autores). A terminologia mais importante para a procura dos estudos sobre TRPE foi "perturbação", que é descrita na literatura de forma imprecisa, porém alguns pesquisadores definem como "treino de perturbação"(JOBGES et al., 2004; SHEN et al., 2012, 2014, 2015). A estimulação mecânica vibratória pode ser descrita como "vibração mecânica localizada", no qual, o estímulo é aplicado no tendão do músculo, ventre muscular ou na orientação das resultantes da força muscular, ou "vibração mecânica generalizada" (Whole Body), estímulo gerado pela plataforma vibratória com efeito generalizado. Além disso, não há títulos nos bancos de dados da literatura descrevendo com precisão assuntos relevantes relacionados à especificidade dos conteúdos que envolvem o TRPE e a EMV em indivíduos com DP.

Esta revisão incluiu estudos não limitados por língua que atendeu aos seguintes critérios: (1) uma metodologia que envolvesse intervenção baseada TRPE ou EVM em pelo menos um grupo, (2) as variáveis de desfecho relacionadas ao 
equilíbrio postural e/ou marcha.

Entraram no espectro de exclusão aqueles estudos que não estavam em conformidade com os critérios de inclusão e de acordo com os limites impostos pela estratégia de busca.

Foram identificados pela estratégia de busca, de acordo com os títulos e resumos, levando à exclusão de alguns estudos por incompatibilidade com os critérios de inclusão estabelecidos. Em seguida, procedeu-se à leitura na íntegra dos estudos selecionados, possibilitando que outros textos fossem igualmente excluídos por não atenderem à proposta da revisão. Em um último momento, as principais informações dos artigos foram sintetizadas para que pudessem orientar as análises descritivas dos estudos selecionados.

\subsubsection{Extração de dados}

Os seguintes dados foram extraídos dos estudos selecionados: identificação da publicação, as características dos participantes (idade, doença, estágio e severidade da doença), características da intervenção experimental e controle (tipo de treinamento, frequência e duração de sessões de treino, duração do programa de treinamento), amostra e principais resultados. Após a análise dos artigos, os dados foram categorizados, interpretados e agrupados de acordo com a similaridade dos dados apresentados.

\subsubsection{Avaliação metodológica}

Os estudos foram avaliados quanto ao nível de qualidade metodológica pela escala PEDro. O objetivo da escala PEDro consiste em auxiliar os utilizadores da base de dados PEDro a identificar rapidamente quais dos estudos clínicos randomizados, ou quase-randomizados, (ECR ou ECC) arquivados na base de dados PEDro poderão ter validade interna (critérios 2-9), e poderão conter suficiente informação estatística para que os seus resultados possam ser interpretados (critérios 10-11) (SHIWA et al., 2011).

\subsection{Resultados}

Como pode ser observado na Figura 1, a estratégia de busca identificou 303 registros de estudos potencialmente relevantes. Destes 303 estudos, 160 foram excluídos por duplicidade, 101 foram excluídos após a leitura de títulos e resumos. Além disso, foram excluídos 26 estudos, com a leitura de textos na íntegra, não apresentaram relação com o estudo proposto, nem com as características estudadas. Uma busca complementar foi realizada, usando o Google Scholar que identificou 125 artigos, os quais foram excluídos por duplicidade. Pelas listas de referências de artigos identificados em revisões pesquisadas para encontrar estudos complementares, 16 estudos foram incluídos nesta revisão, pois relatavam ou TRPE e/ou EMV em indivíduos com DP.

Figura 1 - Resumo da estratégia de pesquisa. A pesquisa inicial nas bases de dados identificou 178 estudos (Medline 143, Pedro 7, SciELO 15 e Cochrane 13 estudos), 125 estudos encontrados no Google Scholar. A maioria dos estudos foi excluída por duplicidade. Selecionados 16 estudos incluídos na análise final
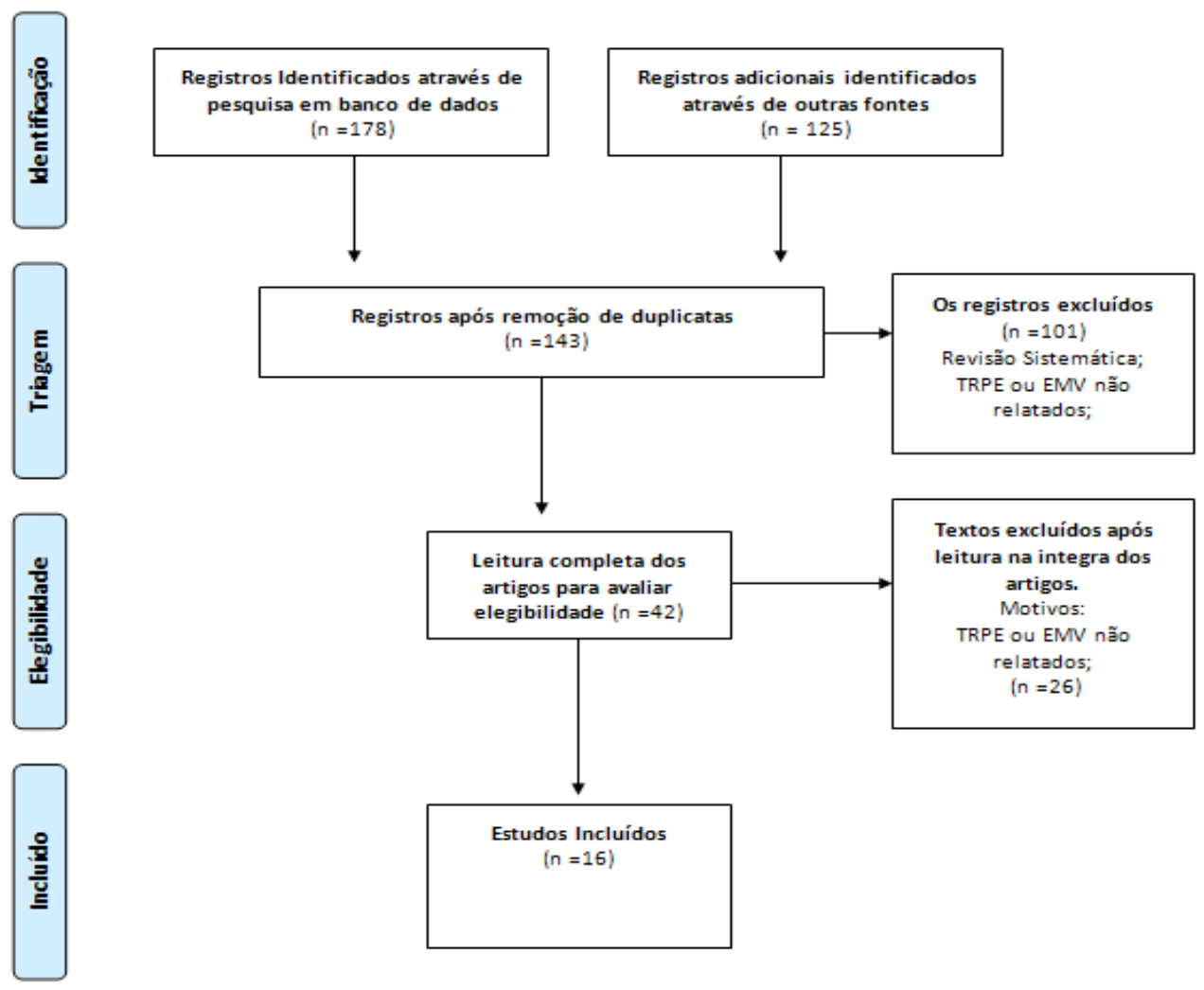

Fonte: Os autores. através de outras fontes ( $n=125$ )
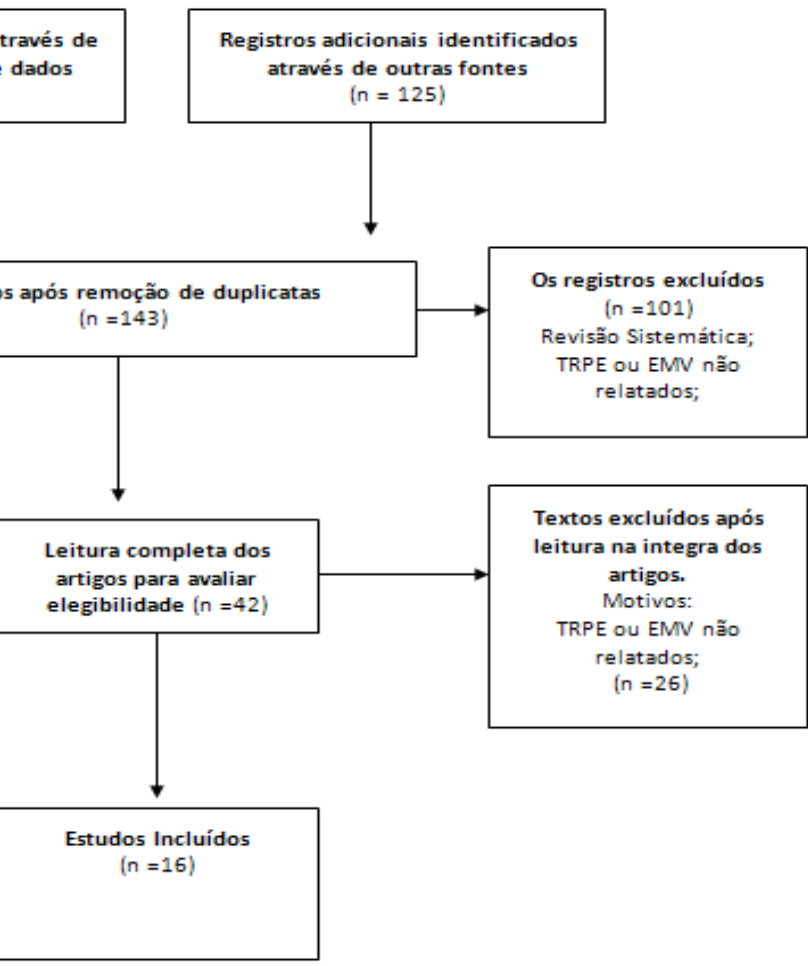


\subsubsection{Características dos estudos}

Esta revisão incluiu 16 estudos com um total de 523 indivíduos, dos quais 265 eram do grupo de intervenção, que envolvia TRPE ou EMV e 258 indivíduos eram do grupo controle. Nos estudos foram incluídos indivíduos com DP de ambos os sexos. A média de idade dos participantes desta revisão no grupo experimental foi de 66,8 anos, desviopadrão de 3.8 e grupo controle de 67,3 anos e desvio-padrão de 6.4. Os estudos foram conduzidos na Alemanha, Brasil, China, Estados Unidos, Itália. A classificação média da doença de Parkinson (Hoen\&Yahr) variou entre os estágios II (Envolvimento bilateral ou linha média sem comprometimento do equilíbrio de forma Bilateral) e III (Doença Bilateral leve a moderada: com deficiência de reflexos posturais; fisicamente independente). $\mathrm{O}$ grupo de controle ativo foi incluído em seis estudos (DE AZEVEDO et al., 2016; SHEN et al., 2012, 2014, 2015; SCHLENSTEDT et al., 2015; WINFREE et al., 2012). Em um estudo a intervenção do grupo controle não foi informada (LANDERS et al., 2016). Em três estudos o grupo controle incluiu exercícios de equilíbrio e/ou mobilidade (CIONCOLONI et al., 2017; DE AZEVEDO et al., 2016; HIGH et al., 2018); em outros três estudos a intervenção de controle incluiu exercícios de fortalecimento de membros inferiores (SHEN et al., 2012, 2014, 2015).

Um estudo de intervenção controle incluiu exercícios de resistência (SCHLENSTEDT et al., 2015); e cinco estudos de intervenção controle incluíram apenas caminhada na esteira (KLAMROTH et al., 2016, PASLUOSTA et al., 2017, STEIB et al., 2017, 2019; WINFREE et al, 2012). No estudo de Volpe et al. (2014) a intervenção do grupo controle incluiu placebo para o dispositivo Equistasi (tecnologia vibratória e que gera vibrações mecânicas de frequência focal constante). Todos os indivíduos foram medicados (estado $\mathrm{ON}$ ) durante o tratamento e a medicação mais utilizada foi a levodopa.

As características da intervenção são descritas de acordo com a frequência, a intensidade, o tempo e o tipo de treinamento de perturbação ou estimulação mecânica vibratória. Seis estudos relataram a frequência das sessões de treinamento três vezes por semana (DE AZEVEDO et al., 2016; KLAMROTH et al., 2016; SHEN et al., 2012, 2014, 2015; VOLPE et al., 2014). Três estudos relataram a frequência de sessões de treinamento 2 vezes por semana (SCHLENSTEDT et al., 2015; STEIB et al., 2017, 2019). O estudo de Klamroth et al. (2015), relatou a frequência de uma sessão de intervenção. O estudo de Volpe et al. (2014), relatou frequência de quatro vezes por semana. Um estudo relatou a frequência de cinco sessões de EMV com duração de 60 segundos (KAMMERMEIER et al., 2017).

Winfree et al. (2012), relataram a frequência de 9 sessões de caminhada (30 metros), com sensores de vibração mecânica no sapato. Em outro estudo os participantes realizaram duas sessões consecutivas, com e sem feedback vibrotátil, com a frequência adaptada para cada condição (HIGH et al., 2018).
Oito estudos relataram a quantidade de perturbações pelo tempo de intervenção de 45 e 60 segundos por sessão e os tempos de 10, 30, 40, 45, 60 minutos por sessão (SHEN et al., 2012, 2014, 2015; VOLPE et al., 2014; KLAMROTH et al., 2016; LANDERS et al., 2016; PASLUOSTA et al., 2017; SCHLENSTEDT et al., 2015). Em cinco estudos as perturbações foram realizadas através de puxões, empurrões ou de forma mecânica com um pêndulo de alumínio gerando perturbações inesperadas nos ombros dos indivíduos com DP em várias direções (SHEN et al, 2012; SCHLENSTEDT et al., 2015; DE AZEVEDO et al., 2016; KLAMROTH et al., 2016; PASLUOSTA et al., 2017).

Em seis estudos as perturbações foram aplicadas, ou por um objeto inesperado, colocado na superfície da esteira ergométrica, ou por paradas bruscas inesperadas (KLAMROTH et al., 2016; PASLUOSTA et al., 2017; SHEN et al., 2014, 2015; STEIB et al., 2017, 2019). No estudo de Cioncoloni et al. (2017), as perturbações foram aplicadas pela plataforma de força. No estudo de Landers et al. (2016) a perturbação externa foi realizada pela inclusão de peso $(5 \mathrm{~kg})$ de forma inesperada.

Em relação aos desfechos relacionados ao controle postural, todos os estudos que encontraram melhora utilizaram escalas de equilíbrio postural como a Berg, ABC e o Mini BEStest (SHEN et al., 2012, 2014, 2015, VOLPE et al., 2014) e plataforma de força (CIONCOLONI et al., 2017; KLAMROTH et al., 2016; LANDERS et al., 2016; STEIB et al., 2017, 2019), que analisaram o centro de pressão com o uso de plataforma de força. Somente Schlentedt et al., (2015), encontraram maior benefício do treinamento de força no controle postural em relação ao TRPE. O estudo de De Azevedo et al. (2016), analisou o centro de pressão com o uso de plataforma de força e o registro da atividade elétrica de um estímulo muscular através da Eletromiografia.

Em apenas três estudos foram relatados o tempo de duração da EMV, que variou de 60 segundos a 30 minutos (KAPUR et al., 2012, WINFREE et al., 2012, KAMMERMEIER et al., 2017). A intensidade de cada EMV variou entre os estudos de $30 \mathrm{~Hz}$ a $500 \mathrm{~Hz}$ (KAPUR et al., 2012, WINFREE et al., 2012, KAMMERMEIER et al., 2017). Dois estudos (KAPUR et al., 2012; HIGH et al., 2018) realizaram a EMV generalizada (WholeBody); e em dois estudos, a EMV foi aplicada de forma localizada (KAMMERMEIER et al., 2017; WINFREE et al., 2012). O tempo de vibração a cada sessão não foi especificado em um estudo (HIGH et al., 2018). As vibrações mecânicas foram geradas por equipamentos específicos, dentre eles, a plataforma vibratória (HIGH et al., 2018), Poltrona Massageadora (KAPUR et al., 2012), EMV associada a tecnologia, chamada PDShoe, que envolve sensores de força e um sistema de vibração que vibra toda vez que o pé de um participante toca o chão (WINFREE et al., 2012) e sensores de vibração no Pescoço (KAMMERMEIER et al., 2017). O estudo de Volpe e col. (2014), realizou perturbação postural com dispositivo Equistasi (tecnologia 
vibratória e que gera vibrações mecânicas de frequência focal constante, aplicadas sobre a $7^{\mathrm{a}}$ vértebra cervical e nos tendões do músculo sóleo). Em relação aos desfechos relacionados ao controle postural, todos os estudos que encontraram melhora utilizaram escalas de equilíbrio postural como a Berg, Teste de Tinetti, teste de caminhada 10 metros, UPDRS III (exame motor), TUG - TimedUpand Go, Pulltest, e escala de instabilidade postural e dificuldade de marcha (KAPUR et al., 2012; KAMMERMEIER et al., 2017; VOLPE et al., 2014; WINFREE et al., 2012) e apenas um estudo que analisou o centro de pressão com o uso de plataforma de força (HIGH et al., 2018).

\subsubsection{Avaliação da qualidade metodológica}

Os escores PEDro dos estudos incluídos variaram de 2 a 8 de um máximo de 10. Em dois estudos os critérios de elegibilidade não foram especificados (SCHLENSTEDT et al., 2015, WINFREE et al., 2012). Quatro estudos não realizaram distribuição aleatória (DE AZEVEDO et al., 2016; CIONCOLONI et al., 2017; HIGH et al., 2018; WINFREE et al., 2012,) e seis estudos não fizeram alocação dos participantes de forma secreta (DE AZEVEDO et al., 2016; CIONCOLONI et al., 2017; HIGH et al., 2018; SHEN et al., 2012; SCHLENSTEDT et al., 2015; WINFREE et al., 2012). Dez estudos incluíram avaliador cego (HIGH et al.,2018; KAPUR et al., 2012; KAMMERMEIER et al., 2017; SHEN et al., 2012, 2014, 2015; SCHLENSTEDT et al., 2015; PASLUOSTA et al., 2017, STEIBET et al., 2017, 2019;). Em três estudos (KLAMROTH et al., 2016; SCHLENSTEDT et al., 2015; WINFREE et al., 2012), as mensurações de pelo menos um resultado-chave não foram obtidas em mais de $85 \%$ dos sujeitos inicialmente distribuídos pelos grupos. Os escores totais de qualidade metodológica variaram de 2 a 8 pontos, sendo que, seis estudos foram classificados como tendo baixa qualidade metodológica (37.5\%) (DE AZEVEDO et al., 2016; HIGH et al., 2018; KLAMROTH et al., 2016; KAMMERMEIER et al., 2017; LANDERS et al., 2016; WINFREE et al., 2012) e 10 estudos foram classificados como tendo moderada e alta qualidade metodológica (CIONCOLONI et al., 2017; KAPUR et al., 2012; SHEN et al., 2012, 2014, 2015; LANDERS et al., 2016; PASLUOSTA et al., 2017; STEIBET et al., 2017, 2019; VOLPE et al., 2014).

\subsection{Discussão}

Os resultados desta revisão fornecem evidências de que o treino para recuperação de perturbação externa - TRPE e a estimulação mecânica vibratória - EMV, assim como verificado previamente em outros estudos (NANHOEMAHABIER et al., 2012; MANSFIELD et al., 2014), são abordagens promissoras na reabilitação do controle postural e marcha em indivíduos com DP. Os resultados mais consistentes da presente revisão indicam que tanto o TRPE, como a EMV, tem o potencial de melhorar alguns domínios do equilíbrio postural e da marcha. No entanto, existem muitas limitações nos estudos incluídos nesta revisão que impedem uma conclusão robusta sobre o efeito da TRPE e a EMV em indivíduos comDP.

Uma dificuldade na generalização dos resultados desta revisão sistemática é a baixa validade externa devido a falta de aleatorização e determinação do grupo controle em alguns estudos (KLAMROTH et al., 2016; HIGH et al., 2018; SHEN et al., 2015; WINFREE et al., 2012;). Como exemplo, verificouse que alguns estudos nem sequer relataram a atividade a qual foi exposto o grupo controle (KLAMROTH et al., 2016; SHEN et al., 2015). Um fator que limita a validade externa é a inclusão de indivíduos somente do estágio moderado (H\&Y= 2 a 3). Além disso, a investigação dos efeitos do TRPE e EMV em outros estágios da doença podem esclarecer o momento ideal de resposta mais efetiva a cada tipo de intervenção.

Mesmo aqueles que utilizaram equipamentos como esteira, mais fáceis de serem programados, não relataram os parâmetros de perturbação postural (KLAMROTH et al., 2016; PASLUOSTA, et al., 2017; SHEN et al., 2012, 2015; STEIB et al., 2017, 2019). Outra limitação importante que prejudica a capacidade de transferência dos benefícios obtidos pelo TRPE é a pouca imprevisibilidade das perturbações aplicadas. Apesar dos participantes não conseguirem prever o momento da perturbação, eles tinham condições de prever a direção e intensidade, o que diminui bastante a capacidade de preparar o indivíduo para perturbações inesperadas do dia a dia. Os pesquisadores não padronizaram os parâmetros de equipamentos como, a plataforma vibratória (EBERSBACH et al., 2008; CHOUZA et al., 2011; HAAS et al., 2006), a cadeira vibratória (KAPUR et al., 2012), sensores e calçado personalizado com estímulo vibratório localizado (KAMMERMEIER et al., 2017; WINFREE et al., 2012). Em um estudo, o parâmetro de intensidade da vibração não foi relatdo (HIGH et al., 2018). Finalmente, tanto a frequência de perturbações, como a intensidade das vibrações mecânicas variaram muito entre os estudos, dificultando uma análise sobre a quantidade de perturbações e a intensidade vibratória ideal para atingir os objetivos da reabilitação.

A presente revisão mostra que a maior parte dos estudos demonstraram melhoras no controle postural após o TRPE e/ou EMV em indivíduos com DP. Em alguns estudos a EMV não provou ser melhor que os resultados obtidos pela intervenção de controle (KAPUR et al., 2012) No estudo de Arias et al. (2009), seus resultados mostraram que o programa de treinamento com vibração, não apresentou diferenças nas escalas de avaliação entre o GE (vibração) e o GC (placebo) em quaisquer desfechos estudados. No entanto, como a maior parte dos resultados utilizou escalas subjetivas de avaliação do controle postural, dificulta a determinação objetiva de quais mecanismos do controle postural são beneficiados. Por exemplo, o Mini-BESTest, utilizado no estudo de Steibet e col. (2017), inclui domínios do controle reativo, antecipatório 
e de integração sensorial. Somente alguns estudos avaliaram o controle postural por meio da biomecânica com plataforma de força que pode gerar dados mais fidedignos do controle postural (HIGH et al., 2018; KLAMROTH et al., 2016; PASLUOSTA et al., 2017; STEIB et al., 2017).

Esta revisão constatou que existe ainda um número reduzido de estudos controlados, aleatorizados, sobre ambas as intervenções, em pacientes com DP, sendo que estes estudos apresentam ainda uma qualidade metodológica variável. Há, portanto, a necessidade da condução de mais estudos com boa qualidade metodológica, a fim de que outras perguntas de pesquisa, como frequência de prática necessária para alcançar adequada redução dos sintomas sejam respondidas afim de contribuir assim para a prática baseada em evidências e prática clínica.

\section{Conclusão}

Com base nos estudos analisados, demonstrou-se que o treino para recuperação deperturbação externa e/ou a estimulação mecânica vibratória, favorecem a melhora nodesempenho da marcha e no tratamento da instabilidade postural em indivíduos com DP.Entretanto, existe um número reduzido de estudos controlados, aleatorizados, sobreambas as intervenções, em pacientes com DP.

\section{Agradecimentos}

Trabalho realizado com apoio da Coordenação de Aperfeiçoamento de Pessoal de Nível Superior - Capes, por meio da concessão de bolsa de pesquisa.

\section{Referências}

ABBRUZZESE, G.; TROMPETTO, C.; MARINELLI, L. The rationale learning in Parkinson's disease. Eur. J. Phys. Rehabil. Med., v.45, p.209-214, 2009

AHLSKOG, J.E. Does vigorous exercise have a neuroprotective effect in Parkinson disease? Neurology, v.77, p.288-294, 2011. doi: https://n.neurology.org/content/77/3/288.

ALLEN, N.E. et al. Balance and falls in Parkinson's disease: a meta-analysis of the effect of exercise and motor training. Mov. Disord., v.26, n.9, p.1605-1615, 2011. doi: https://doi. org/10.1002/mds.23790.

ANDRADE, L.A.F. Contribuição ao estudo da doença de Parkinson de início precoce: análise clínica de 58 pacientes. São Paulo: Universidade Federal de São Paulo, 1994.

ARIAS, P.; CHOUZA, M.; VIVAS, J. Effect of whole body vibration in Parkinson's Disease: a controlled study. Mov. Disord., v.24, n.6, p.891-898, 2009. doi: https://doi.org/10.1002/ mds. 22468 .

CHOUZA, M. et al. Acute effects of whole-body vibration at 3,6 , and $9 \mathrm{hz}$ on balance and gait in patients with Parkinson's disease. Mov. Disord., v.26, n.5, p.920-921, 2011 doi: 10.1002/ mds. 23582 .

CIONCOLONI, D. et al. Impaired scaling of preparatory postural responses to repeated balance perturbations in Parkinsonian patients with comorbid white matter disease. Neuro Rehabilitation, v.40, n.3, p.421-427, 2017
COUTO, B.P. et al. Chronic effects of different frequencies of local vibrations. Int. J. Sports Med., v.33, p.123-129, 2012.

CRENNA, P. et al. Impact of subthalamic nucleus stimulation on the initiation of gait in Parkinson's disease. Experimental Brain Res., v.172, n.4, p.519-532, 2006.

DE AZEVEDO A. et al. Anticipatory and compensatory postural adjustments subjects with Parkinson's Disease. Plos One, v.11, n.5 E0155012, 2016. doi: https://doi.org/10.1371/journal. pone. 0155012 .

DE NUNZIO, A.M. et al. Alternate rhythmic vibratory stimulation of trunk muscles affects walking cadence and velocity in Parkinson's Disease. Clin. Neurophysiol., v.121, p.240-247, 2010. doi: https://doi.org/10.1016/j.clinph.2009.10.018.

DRUMMOND, M.D.M. Efeito da aplicação de vibração mecânica localizada durante o treinamento de força sobre a hipertrofia muscular. Belo Horizonte: Universidade Federal de Minas Gerais, 2012.

EBERSBACH, G. et al. Whole body vibration versus conventional physiotherapy to improve balance and gait in Parkinson's Disease. Arch. Phys.. Med. Rehabil., v.89, p.399-403, 2008. doi: https:// doi.org/10.1016/j.apmr.2007.09.031.

GEORGE, S.T. et al. Compensatory stepping in Parkinson's Disease is still a problem after deep brain stimulation randomized to Stn or Gpi. J. Neurophysiol., v. 2015;114(3):1417-1423, 2015. doi: 10.1016 / j.apmr.2007.09.031.

HAAS, A. et al. Proprioceptive and sensorimotor performance in Parkinson's Disease. Res. Sports Med., v.14, n.4, p. 273-287, 2006. doi: $10.1080 / 15438620600985902$.

HELY, M.A. et al. The Sydney multicenter study of Parkinson's disease: the inevitability of dementia at 20 years. Movement Dis., v.23, n.6, p.837-844, 2008. doi: https://doi.org/10.1002/ mds. 21956 .

HENSCH, T. Critical period plasticity in local cortical circuits. Nat. Rev. Neurosc., v.6, n.11, p.877-888, 2005. doi: 10.1038 / nrn 1787

HIGH, C.M. et al. Vibrotactile feedback alters dynamics of static postural control in persons with parkinson's disease but not older adults at high fall risk. Gait Posture, 2010. doi: https://doi. org/10.1016/j.gaitpost.2018.05.010.

HIRSCH, M. et al. The effects of balance training and highintensity resistance training on persons with idiopathic Parkinson's disease. Arch. Phys. Med. Rehab., v.84, n.8, p.1109-1117, 2003. doi: https://doi.org/10.1016/S0003-9993(03)00046-7.

JANKOVIC, J. Parkinson's disease: clinical features and diagnosis. J. Neurol. Neurosurg. Psychiatry, v.79, p.368-376, 2008. doi: 10.1136 / jnnp.2007.131045.

JOBGES, M. Repetitive training of compensatory steps: a therapeutic approach for postural instability in Parkinson's disease. J. Neurol. Neuros. Psychiatry, v.75, n.12, p.1682-1687, 2004. doi: 10.1136/jnnp.2003.016550.

KAMMERMEIER, S. et al. Neck vibration proprioceptive postural response intact in progressive supranuclear palsy unlikeidiopathic Parkinson's Disease. Front. Neurol., v.8, p.689, 2017. doi: https://doi.org/10.3389/fneur.2017.00689.

KAPUR, S.S.; KAPUR, G.T.; GOETZ, C.G. Vibration therapy for Parkinson's Disease: charcot's studies revisited. J. Parkinsons Dis., v.2, n.1, p.23-27, 2012. doi: 10.3233/JPD-2012-12079.

KHUDADOS, E.; CODY, F.W.; O’BOYLE, D.J. Proprioceptive regulation ofvoluntary ankle movements, demonstrated using muscle vibration,is impaired by Parkinson's disease. J. Neurol. Neurosurg. Psychiatry, v.67, p.504-510, 1999. doi; https://jnnp. 
bmj.com/content/67/4/504.long.

KING, L.K.; ALMEIDA, Q.J.; AHONEN, H. Shortterm effects of vibration therapy on motor impairments in Parkinson's disease. Neuro Rehabil., n.25, p.297-306, 2009, doi 10.3233/NRE-20090528

KITADA, T. et al. Mutations in the parkin gene cause autosomal recessive juvenile parkinsonism. Nature, v.392, n.6676, p.605608, 1998. doi: $10.1038 / 33416$.

KLAMROTH, S.Effects of exercise therapy on postural instability in Parkinson Disease: a meta-analysis. J. Neurol. Phys. Ther., v.40, n.1, p.3-14, 2016. doi: 10.1097/NPT.0000000000000117.

KLOCKGETHER, T.M. et al. A defect of kinesthesia in Parkinson's disease. Mov. Disord, n.10, p.460-465, 1995. doi: https://doi.org/10.1002/mds.870100410.

LAESSOE, U.; VOIGT, M.Anticipatory postural control strategies related to predictive perturbations. Gait Posture, v.28, n.1, p.6268, 2008. doi: https://doi.org/10.1016/j.gaitpost.2007.10.001.

LANDERS, M. et al. Does attentional focus during balance training in people with Parkinson's disease affect outcome? A randomised controlled clinical trial. Clin. Rehabil., v.30, n.1, p.53-63, 2016. doi: https://doi.org/10.1177/0269215515570377.

LAUREN, K. et al. Short-term effects of vibration therapy on motor impairments in Parkinson's disease. J. Parkinson's Disease, n.2, p.23-27, 2012. doi 10.3233/JPD-2012-12079.

LEE, R. et al. Preparatory postural adjustments in parkinsonian patients with postural instability. Can. J. Neurol. Sci., v.22, n.2, p.126-135, 1995. doi: 10.1017/s0317167100040208.

LENT, R. Cem bilhões de neurônios. Rio de Janeiro: Atheneu, 2010.

LIBERATI, A. et al. The PRISMA statement for reporting systematic reviews and meta-analyses of studies that evaluate health care interventions: explanation and elaboration. PLoS Med., v.6, n.7, e1000100, 2009. doi: https://doi.org/10.1371/ journal.pmed.1000100.

MAK, M. et al. Long-term effects of exercise and physical therapy in people with Parkinson disease. Nat. Rev Neurol., v.13, n.11, p.689-703, 2017. doi: 10.1038/nrneurol.2017.128.

MAKI, B.E.; MCILROY, W.E. Change-in-support balance reactions in older persons: an emerging research area of clinical importance. Neurol. Clin., v.23, p.751-783, 2005. doi: https://doi. org/10.1016/j.ncl.2005.01.002

MANCINI, M. et al. Effects of Parkinson's disease and levodopa on functional limits of stability. Clin. Biomechanics, v.23, n.4, p.450-458, 2008. doi: 10.1016/j.clinbiomech.2007.11.007.

MANSFIELD, A. et al. Does perturbation-based balance training prevent falls? systematic review and meta-analysis of preliminary randomized controlled trials. Phys. Ther., v.95, n.5, p.700-709, 2014. doi: https://doi.org/10.2522/ptj.20140090.

MORRIS, M.E. et al. Striding out with parkinson disease: evidence-based physical therapy for gait disorders. Phys. Ther., v.90, n.2, 2010. doi: https://doi.org/10.2522/ptj.20090091.

NANHOE-MAHABIER, W. et al. BloemBr.the effects of vibrotactile biofeedback training on trunk sway in Parkinson's disease patients. Parkinsonism Relat Disord., v.18, p.1017-1021, 2012. doi: https://doi.org/10.1016/j.parkreldis.2012.05.018.

PARK, J.; KANG, Y.; HORAK, F. What is wrong with balance in Parkinson's Disease? J. Movement Dis., v.8, n.3, p.109-114, 2015. doi: 10.14802/jmd.15018.

PASLUOSTA, C. et al. Acute neuromuscular adaptations in the postural control of patients with Parkinson's Disease after perturbed walking. Frontiers Aging Neurosc., n.9, 2017. doi: $10.3389 /$ fnagi.2017.00316.

PETERSON, D. et al. Cognitive contributions to freezing of gait in parkinson disease: implications for physical rehabilitation. Phys. Ther., v.96, n.5, p.659-670, 2015. doi: 10.2522/PTJ.20140603.

PROTAS, E.J. et al. Gait and step training to reduce falls in Parkinson's disease. Neuro Rehabil., v.20, p.183-190, 2005. doi: 10.3233/NRE-2005-20305.

SCHLENSTEDT, C. et al. Resistance versus balance training to improve postural control in parkinson's disease: a randomized rater blinded controlled study. Plos One, v.10, n.10, 2015. doi: https://doi.org/10.1371/journal.pone.0140584.

SCHONEBURG, B. et al. Framework for understanding balance dysfunction in Parkinson's disease. Movement Dis., v.28, n.11, p.1474-1482, 2013. doi: https://doi.org/10.1002/mds.25613.

SHEN X, Mak M. Repetitive step training with preparatory signals improves stability limits in patients with Parkinson disease. J. Rehabil. Med., v.44, n.11, p.944-949, 2012. doi: $10.2340 / 16501977-1056$.

SHEN, X.; MAK, M. Technology-assisted balance and gait training reduces falls in patients with parkinson's disease. Neurorehabil. Neural Repair, v.29, n.2, p.103-111, 2014.

SHEN, X.; MAK, M.K.Y. Technology-assisted balance and gait training reduces falls in patients with Parkinson's disease: a randomized controlled trial with 12-month follow-up. Neurorehabil. Neural Repair., v.29, p.103-111, 2015. doi: https:// doi.org/10.1177/1545968314537559.

SHIWA, S. et al. PEDro: a base de dados de evidências em fisioterapia. Fisioter. Mov., v.24, n.3, p.523-533, 2011. doi: https://doi.org/10.1590/S0103-51502011000300017

SILVA, J.D.; COLETTA, D.V.M.; FEN, H.C. Transtornos do movimento: diagnóstico e tratamento. São Paulo: Academia Brasileira de Neurologia, 2016.

SMANIA, N. et al. Effect of balance training on postural instability in patients with idiopathic Parkinson's Disease. Neurorehabil. Neural Repair, v.24, n.9, p.826-834, 2010. doi: 10.1177 / 1545968310376057.

STEIB, S. et al. Perturbation during treadmill training improves dynamic balance and gait in parkinson's disease: a single- blind randomized controlled pilot trial. Neurorehabil. Neural Repair, v.31, n.8, p.758-768, 2017. doi: 10.1177 / 1545968317721976.

STEIB, S. et al. Exploring gait adaptations to perturbed and conventional treadmill training in Parkinson's disease: Timecourse, sustainability, and transfer. Hum. Mov. Sci., v.64, p.123132, 2019. doi: https://doi.org/10.1016/j.humov.2019.01.007.

TAJIRI, N. et al. Exercise exerts neuroprotective effects on parkinson's disease model of rats. Brain Res., v.1310, p.200-207, 2010. doi: https://doi.org/10.1016/j.brainres.2009.10.075.

TOOLE, T. et al. The effects of a balance and strength training program on equilibrium in Parkinsonism: a preliminary study. Neuro Rehabil., v.14, p.165-174, 2000. doi: 10.3233/NRE-200014306.

TSOU, Y.H. et al. Treadmill exercise activates Nrf2 tioxidant system to protect the nigrostriatal dopaminergic neurons from MPP1toxicity. Exp. Neurol., v.263, p.50-62. 2015. doi: https:// doi.org/10.1016/j.expneurol.2014.09.021.

TURBANSKI, S. et al. Effects of random whole-body vibration on postural control in Parkinson's disease. Res. Sports Med., v.13, p.243-256, 2005. doi: 10.1080/15438620500222588. 
VALKOVIC, P.; KRAFCZYK, S,; BOTZEL, K. Postural reactions to soleus muscle vibration in Parkinson's Disease: scaling deteriorates as disease progresses. Neurosci Lett., v.40, p.92-96, 2006. doi: https://doi.org/10.1016/j.neulet.2006.02.073.

VOLPE, D.; GIANTIN, M.; FASANO, A. A wearable proprioceptive stabilizer (Equistasi ${ }^{\circledR}$ ) for rehabilitation of postural instability in Parkinson's Disease: a phase II randomized double-blind, double-dummy, controlled study. PLoSONE, v.9, n.11, 2014. doi: 10.1371/journal.pone.0112065.

WINFREE, K.N.I. et al. An untethered shoe with vibratory feedback for improving gait of parkinson's patients: the PDShoe, in In proc. of the Conf. of the IEEE on Eng. in Med. and Biology Society, EMBC, 2012, p. 1202-1205. doi: 10,1109/ embc.2012.6346152. 